Brazilian Journal

of Chemical

ISSN 0104-6632

Engineering

\title{
MULTIVARIABLE OPTIMAL CONTROL OF A HEAT EXCHANGER NETWORK WITH BYPASSES
}

\author{
F. Delatore ${ }^{1 *}$, L. F. Novazzi ${ }^{3}$, F. Leonardi ${ }^{2}$ and J. J. da Cruz ${ }^{4}$ \\ ${ }^{1}$ Department of Electrical Engineering, FEI University, Av. Humberto de Alencar \\ Castelo Branco 3972, Zip Code: 09850-901, Sao Paulo - SP, Brazil. \\ Phone: +55 1143532900 \\ E-mail: fdelatore@fei.edu.br \\ ${ }^{2}$ Department of Mechanical Engineering, FEI University, Sao Paulo - SP, Brazil. \\ ${ }^{3}$ Department of Chemical Engineering, FEI University, Sao Paulo - SP, Brazil. \\ ${ }^{4}$ Department of Telecommunications and Control, University of Sao Paulo, Sao Paulo - SP, Brazil.
}

(Submitted: June 18, 2013 ; Revised: December 23, 2014 ; Accepted: February 2, 2015)

\begin{abstract}
Heat exchanger networks present an interesting control problem due to coupling among process streams. In this work, the linear quadratic regulator (LQR), a feedback optimal control technique, is used to control stream temperatures on a laboratory scale heat exchanger network, through bypass manipulation, in a multivariable system. The LQR design was based on a mathematical model of the plant and its performance was compared to traditional PID control and to dynamical decoupling. Experimental tests were performed to evaluate the controllers, involving regulatory and servo problems. The performance of the different controllers was quantitatively compared by using the integral absolute error. Although LQR is not a new control methodology, the results obtained in this work suggest that LQR is an interesting alternative to control HEN when compared to the PID and to the dynamic decoupler. Moreover, one of the main advantages of the LQR is its tuning simplicity, since only one parameter is sufficient for this application.

Keywords: Heat exchanger network; Optimal control; LQR.
\end{abstract}

\section{INTRODUCTION}

Due to the oil price rise since the seventies and to environmental issues, efficient use of energy in chemical processes is very important. Almost thirty years ago the theoretical foundations of Process Integration for the efficient use of energy were established, with Pinch Technology, an elegant approach to set energy / cost targets for heat exchanger networks (HEN), as well as rules to design such networks (Linhoff et al., 1982). Nowadays these synthesis techniques, including some which are based on mathematical programming, are well established in Process
Design and are easily found in many Chemical Engineering textbooks.

The design of a HEN depends on nominal stream supply temperatures and flowrates. However, during plant operation such nominal operating conditions can change, influencing stream target temperatures and propagating in the network, since the heat exchangers introduce coupling among different parts of the process. Therefore, HEN control is an interesting issue and has been addressed in the literature since the eighties. A preliminary important contribution can be found in Marselle et al. (1982), where the authors proposed a HEN design technique by consid-

*To whom correspondence should be addressed 
ering process operability and controllability, which they called process resilience. Operability is defined as the ability of the network to remain steady-state feasible when subjected to process disturbances, whereas controllability is regarded as the network capacity to go from one steady-state to a different one, in a finite time.

Later on, Calandranis and Stephanopoulos (1988) proposed a sequence of control actions of the loops in a network to solve the regulatory and servo control problems in a HEN, exploiting its structural characteristics. The idea of the strategy was the identification of routes through the HEN that could allocate disturbances or setpoint changes to available sinks, i.e., utility heat exchangers.

Based on a previous work on HEN control by Mathisen (1994), Glemmestad et al. (1996) applied a method for optimal operation of the network and studied the coupling of manipulated variables, represented by bypasses positions on the exchangers, with controlled variables, represented by stream target temperatures. In addition to the input / output pairing in the suggested decentralized control scheme, the proposed approach also contemplated the optimization of utility consumption in the HEN, since the number of manipulated variables is greater than the number of controlled parameters, which resulted in a positive degree of freedom. More recently and due to this positive degree of freedom, Sun et al. (2013) used non-square relative gain arrays to choose which bypasses should be selected to control a HEN.

Glemmestad et al. (1999) presented an alternative approach to the optimal operation of HEN systems based on on-line optimization of a steady state function and a fixed control structure. Later, Giovanini and Marchetti (2003) showed that a low-level Distributed Control System is also capable of handling HEN control problems when a flexible control loop structure is provided.

In the work of Lersbamrungsuk et al. (2008), a linear programming (LP) problem for the optimal operation of a HEN was formulated. As a consequence of the LP problem, the optimal point of operation of a HEN remained at some of its constraints. The authors also proposed an offline strategy to switch between active constraints, identifying possible operational regions, and combined this with decentralized control. Previously, Aguilera and Marchetti (1998) developed a procedure for optimization and control of a HEN, in a more complex approach than the latter, since a nonlinear programming problem (NLP) needs to be solved online, during operation.

From the point of view of performance, there are a lot of different control techniques that could be used in heat exchanger networks. These techniques range from methodologies that have a complex and highly engineered design, typically with a superior performance, down to methodologies that are easy and effortless to design, but normally not capable to lead to a desirable performance. As an example, the PID is the most common controller due to its straightforward design procedure and easy implementation.

Nonetheless, in multivariable control problems, as is the case of a HEN, the PID design will demand a supplementary engineering effort to tune well the controllers. In this situation, model predictive control (MPC) may be considered to be a suitable control strategy to be used within industrial process, as it can deal with multivariable systems, complex dynamics and constraints on input and/or output variables. In this approach the future moves of the manipulated variables depend on the model and on plant measured output variables, in such a way that an on-line constrained optimization is performed.

Gonzalez et al. (2006) presented an application of optimization and control of heat exchanger networks, through a two-level control structure. In the lower level, a constrained MPC was used and the higher level was supervised by an online optimizer. The MPC was based on a linear approximate plant model whereas the optimizer was based on a rigorous model. By using a moving horizon, hard constraints on the manipulated variables were dealt with in a straightforward way. Although the proposed methodology uses a consolidated control strategy, just simulated results were presented. Besides, modeling errors were not explicitly included in the design.

In the range of suitable controllers for the control of a HEN, one can also point out the Linear Quadratic Regulator (LQR), which has a very simple design in the time domain. During the design of a LQR, the expected dynamic response is explicitly not taken into account, and the closed-loop response is checked afterwards. If the time response does not present an acceptable performance, it is possible to try a new controller by changing the penalty matrices, resulting in an extraordinary ease to design, and normally a good performance (Delatore et al., 2009).

In order to reduce the difficulties involved in controller design, in this work a control solution for a HEN based on optimal linear control is proposed. The LQR controller performance was successfully checked by experimental results obtained in a pilot plant. It must be pointed out that LQR control applied to a HEN is not commonly found in the scientific literature (Delatore et al., 2009). The objective of this work is to illustrate the proposed design procedure, as well as to show the reasonable perfor- 
mance found for the LQR control to perform the closed loop control on a HEN.

\section{THE MATHEMATICAL MODEL}

The shell and tube heat exchangers (HE) are the most common HE used in chemical plants due to some particular characteristics, mainly associated with their low cost and uncomplicated repair. The dynamic model of a shell and tube exchanger can be written based on the energy balances for hot and cold streams, as in Equations 1 and 2, respectively (Luyben, 1990). In these equations, the subscripts $H$ and $C$ stand for the hot and cold sides, respectively, while $T$ is the stream temperature, $t$ is the time, $m$ is the stream flowrate, $\rho$ its density, $C_{p}$ is the stream specific heat, $z$ is the axial position, $U$ is the overall heat transfer coefficient, $A$ is the heat transfer area, $V$ is the volume and $v$ is a ratio between volume and tube length.

$$
\begin{aligned}
& \frac{\partial T_{H}}{\partial t}=-\frac{m_{H}}{\rho_{H} v_{H}} \frac{\partial T_{H}}{\partial z}-\frac{U A}{\rho_{H} V_{H} C_{p, H}}\left(T_{H}-T_{C}\right) \\
& \frac{\partial T_{C}}{\partial t}=\frac{m_{C}}{\rho_{C} v_{C}} \frac{\partial T_{C}}{\partial z}+\frac{U A}{\rho_{C} V_{C} C_{p, C}}\left(T_{H}-T_{C}\right)
\end{aligned}
$$

Equations (1) and (2) were discretized through the finite difference method, resulting in Equations (3) and (4), where the subscript $i$ is connected to a discretized temperature and $n$ is the number of discretization cells used, where $i=1,2, . ., n$.

$$
\begin{aligned}
& \frac{d T_{H, i}}{d t}=\left(a_{1}-a_{2}\right) T_{H, i-1}-\left(a_{1}+a_{2}\right) T_{H, i} \\
& +a_{2} T_{C, n-i}+a_{2} T_{C, n-i+1} \\
& \frac{d T_{C, n-i+1}}{d t}=a_{4} T_{H, i-1}+a_{4} T_{H, i}+\left(a_{3}-a_{4}\right) T_{C, n-i} \\
& -\left(a_{3}+a_{4}\right) T_{C, n-i+1}
\end{aligned}
$$

In addition to Equations (1) and (2), the dynamical behavior of stream bypasses in the heat exchanger was also modeled and discretized, which resulted in Equations (5) and (6), where the subscript by indicates bypass and $f$ is the bypass fraction.

$\frac{d T_{H b y, i}}{d t}=a_{5} T_{H b y, i-1}-a_{5} T_{H b y, i}$

$$
\frac{d T_{C b y, n-i+1}}{d t}=a_{6} T_{C b y, n-i}-a_{6} T_{C b y, n-i+1}
$$

where,

$$
\begin{aligned}
& a_{1}=n m_{H}\left(1-f_{H}\right) /\left(\rho_{H} V_{H}\right), a_{2}=U A /\left(2 \rho_{H} V_{H} C_{p, H}\right), \\
& a_{3}=n m_{C}\left(1-f_{C}\right) /\left(\rho_{C} V_{C}\right), \\
& a_{4}=U A /\left(2 \rho_{C} V_{C} C_{p, C}\right), \\
& a_{5}=n m_{H} f_{H} /\left(\rho_{H} V_{H b y}\right), \quad a_{6}=n m_{C} f_{C} /\left(\rho_{C} V_{C b y}\right) .
\end{aligned}
$$

Thus a HEN can be mathematically represented by the interconnection of HE, according to the HEN structure. The discretization scheme is represented in Figure 1 for the first, $i$-th and $n$-th stages, respectively (Novazzi, 2007). The system of ordinary differential equations, constituted by $4 n$ equations, was solved numerically.

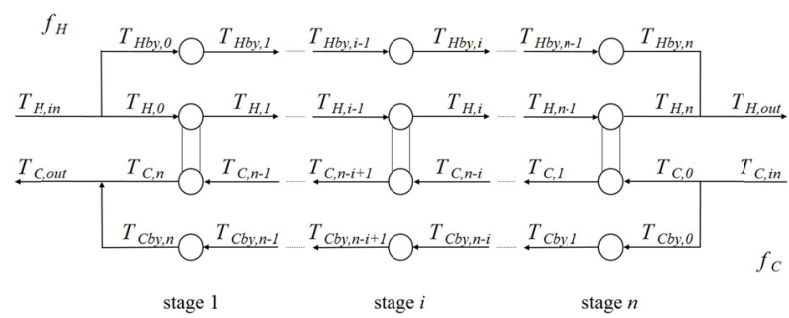

Figure 1: Discretized heat exchanger model.

\section{THE CONTROLLER CHARACTERISTICS}

Classical control techniques for single input single output (SISO) systems are based on analytical tools, such as Laplace transforms, Routh criterion, etc., as well as on graphical tools, such as Bode and Nichols plots. Nevertheless, for the controller design of Multiple Input Multiple Output (MIMO) systems, the use of such techniques may be troublesome. Modern control design techniques based on a statespace model are generally more suitable for MIMO systems (Levine, 2010). In this work, the LQR framework is applied to design the controller in an experimental heat exchanger network. Disturbance attenuation corresponding to inlet temperatures are considered as part of the performance specifications; furthermore, stream outlet temperatures must follow step changes in the setpoints with small steady-state errors and with a smooth transient response, which does not cause actuators saturation.

It is important to compare the proposed approach in relation to other typical control alternatives, such as the dynamic decoupler and classical PID control, so that one can verify if the LQR is also a good 
choice. In the next three sections, a short description of these typical alternatives and of LQR is presented.

\section{a) Dynamic Decoupler}

In multivariable systems, the input and output variables are typically coupled. The direct coupling is the influence of the input $u_{i}$ on its corresponding output $y_{i}$. The cross-coupling is the influence of the input $u_{j}$ on other outputs, rather than the output directly related to it. The direct coupling is the desired form of the couplings that appear in systems since it allows it to be controlled in a simpler form (Ogunnaike and Ray, 1994). The difficulty is found in systems with cross-coupling, because they cause interactions in control loops.

The technique of variable decoupling aims to reduce the effects of existing cross-couplings among variables, allowing the tuning of the controllers to be made in a decentralized manner by scalar controllers. The decoupling can be achieved by adding a proper compensating transfer matrix between the controller output and the input of the plant, as depicted in Figure 2 (Ogunnaike and Ray, 1994).

Based on Figure 2, one can write:

$$
\begin{aligned}
Y_{1}(s)= & \left(G_{11}+G_{12} G I_{2}\right) U_{1}(s) \\
& +\left(G_{11} G I_{1}+G_{12}\right) U_{2}(s) \\
Y_{2}(s) & =\left(G_{21}+G_{22} G I_{2}\right) U_{1}(s) \\
& \partial+\left(G_{21} G I_{1}+G_{22}\right) U_{2}(s)
\end{aligned}
$$

Let the transfer functions $G I_{1}$ and $G I_{2}$ be:

$$
\begin{aligned}
& G I_{1}=-\left(\frac{G_{12}}{G_{11}}\right) \\
& G I_{2}=-\left(\frac{G_{21}}{G_{22}}\right)
\end{aligned}
$$

The new transfer functions of the system, now without the interactions between the variables, are:

$$
\begin{aligned}
& Y_{1}(s)=\left[G_{11}-\left(\frac{G_{12} G_{21}}{G_{22}}\right)\right] U_{1}(s) \\
& Y_{2}(s)=\left[G_{22}-\left(\frac{G_{12} G_{21}}{G_{11}}\right)\right] U_{2}(s)
\end{aligned}
$$

Although the technique theoretically is able to completely eliminate interactions among variables, in practice this decoupling may not occur completely, since the technique is based on a process model, which is not an exact representation of reality. The decoupling system is usually not used on a stand-alone basis. After applying the decoupler, a scalar design technique is normally used to design a PID controller.

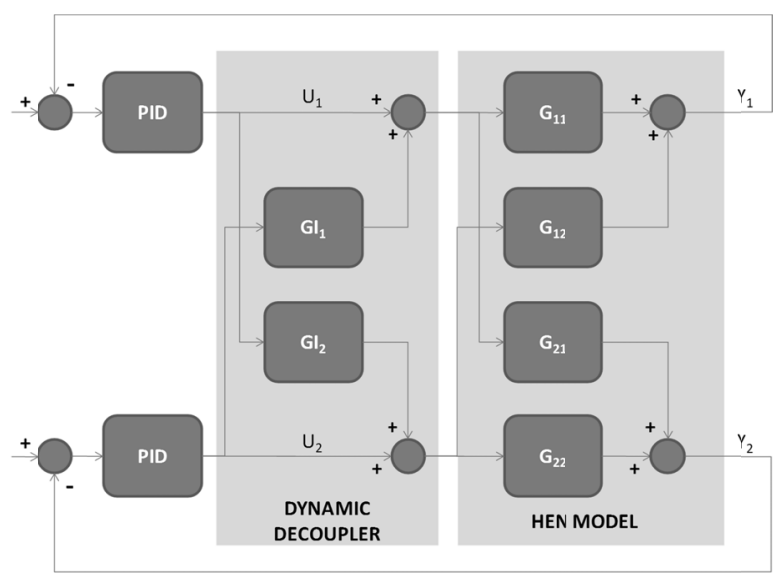

Figure 2: Dynamic decoupling system.

\section{b) PID Control}

When subjected to bypass manipulation, the stream outlet temperature in a heat exchanger connected directly to this bypass presents a lead lag response with a small time delay (Novazzi, 2007). Rotea and Marchetti (1997) showed that integral control is the best option to control the temperature in a heat exchanger with bypasses, as indicated in Equation (13), where $g_{C}$ is the controller transfer function and $K_{I}$ is the integral gain.

$g_{C}(s)=\frac{K_{I}}{s}$

These authors proposed a tuning formula for the integral controller that depends only on the initial temperature jump $K_{1}$ of a step response in the bypass and on process dead time $\alpha_{d}$, as follows:

$K_{I}=\frac{0.739}{K_{1} \alpha_{d}}$

\section{c) LQR Control}

Let the system be given in state-space form as: 
$\frac{d}{d t} x(t)=A x(t)+B u(t)$

$y(t)=C x(t)$

where $x$ is the state vector, $u$ is the input vector, $y$ is the output vector and $A, B, C$ are constant matrices with appropriate dimensions. The optimal control law that minimizes the performance index:

$J=\int_{0}^{\infty}\left[x(t)^{T} Q x(t)+u(t)^{T} R u(t)\right] d t$

where $Q=Q^{T} \geq 0$ and $R=R^{T}>0$ are, respectively, the state and control weighting matrices, is given by the linear state feedback:

$u(t)=-K x(t)$

forming the so-called linear quadratic regulator (Levine, 2010). The state feedback matrix $K$ is given by:

$K=R^{-1} B^{T} P$

where $P=P^{T} \geq 0$ is the solution of the following Riccati Algebraic Equation:

$A^{T} P+P A-P B R^{-1} B^{T} P+Q=0$

Since the LQR ensures the minimization of a cost function regardless of the $Q$ and $R$ values, the solution is always optimal in the mathematical sense. Thus, they are the tuning parameters of the design. These matrices impose penalties on the states and controls and can be used in diagonal form, i.e., without a penalty cross between variables. If one wants to give importance only to the states associated with the outputs $y(t)$ and their integral $z(t)$, it is possible to rewrite the matrix $Q$ as:

$Q=\left[\begin{array}{cc}C^{T} Q_{y} C & 0 \\ 0 & Q z\end{array}\right]$

such that the variables $y$ and $z$ are explicitly included in the cost function, where $Q_{\mathrm{y}}$ penalizes the outputs and $Q_{\mathrm{z}}$ penalizes the integral of the outputs. Thus the cost function can be written as:

$J=\int_{0}^{\infty}\left(y^{T} Q_{y} y\right)+\left(z^{T} Q_{z} z\right)+\left(u^{T} R u\right) d t$
In this work, an identical penalty is proposed for all states to prevent saturation of any internal state. Due to the symmetry of the problem and since the values have the same characteristics, controls were equally penalized too. By appropriately choosing the penalties, which in this case means to find just an unique constant value as a common factor in the $Q$ matrix, not only can a desired time response generally be obtained, but also a satisfactory tradeoff between speed of response and control effort. If the state vector cannot be fully measured, the feedback loop using the LQR gain matrix $K$ can be built using a state observer as shown in Figure 3, where $x_{S P}$ are the setpoints for all states.

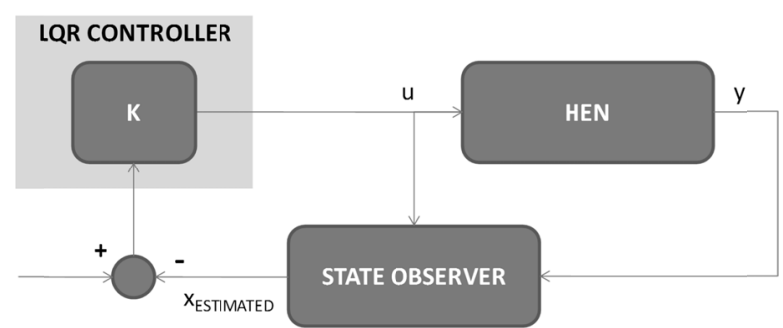

Figure 3: Feedback system with state observer and state setpoints included in the loop.

A more common situation occurs when setpoints are defined not for the states $x$ but for the output vector $y$. In this way a modified structure can be used, where the error between the setpoint and the plant output is integrated in order to increase the gain at low frequencies, as shown in Figure 4 (Delatore et al., 2009; Delatore et al., 2010).

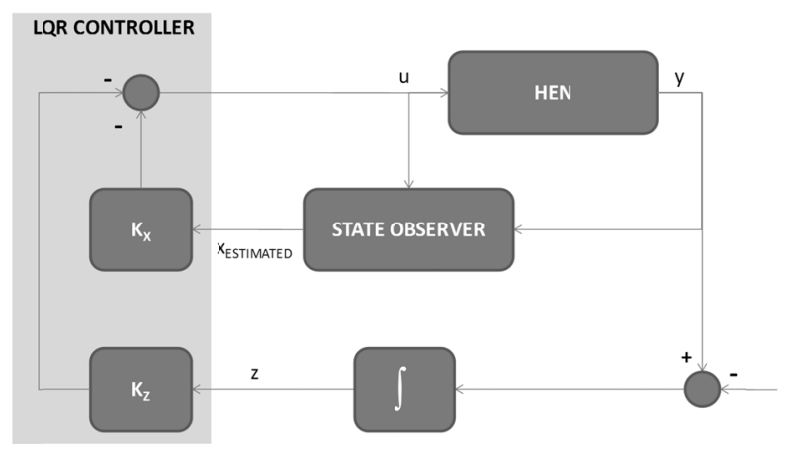

Figure 4: Feedback system with state observer, output setpoints and integrator block included in the loop.

Extra states are thus added to the system, giving rise to an extended state $x_{a}$ :

$x_{a}(t)=\left[\begin{array}{l}x(t) \\ z(t)\end{array}\right]$ 
where:

$$
\frac{d}{d t} z(t)=y(t)=C x(t)
$$

The state feedback matrix $K_{a}$ can thus be correspondingly partitioned as:

$$
K_{a}=\left[\begin{array}{ll}
K_{x} & K_{z}
\end{array}\right]
$$

By taking into account Equations (22) and (23), the state model in Equation (15) can be rewritten as:

$$
\frac{d}{d t} x_{a}(t)=A_{a} x_{a}(t)+B_{a} u(t)
$$

where:

$$
\begin{aligned}
A_{a} & =\left[\begin{array}{ll}
A & 0 \\
C & 0
\end{array}\right] \\
B_{a} & =\left[\begin{array}{l}
B \\
0
\end{array}\right]
\end{aligned}
$$

Thus, the augmented matrices $A_{a}$ and $B_{a}$ are the new matrices used in the LQR project.

\section{METHODOLOGY}

Before presenting the control design and its performance applied to a HEN, it is important to define the physical arrangement of the exchangers, determining the manipulated, the controlled and the disturbance variables of the experimental network considered in this work, as shown in Figure 5 in a grid diagram.

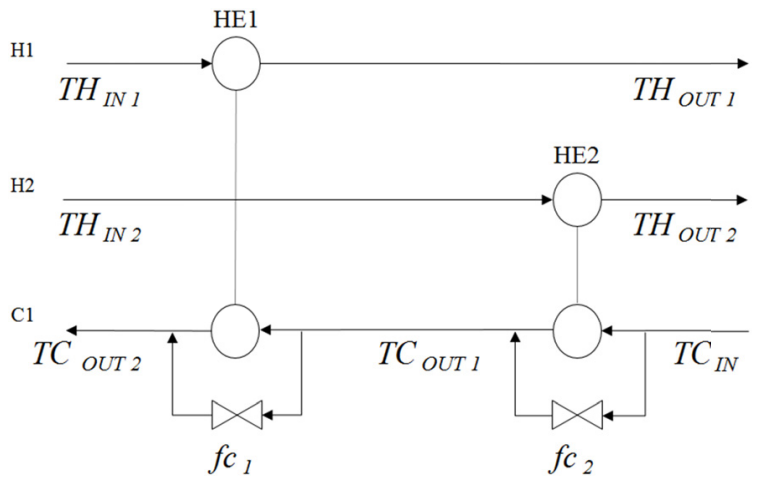

Figure 5: The HEN basic structure.
The experimental HEN is constituted by two heat exchangers (HE1 and HE2) and has two hot (H1 and $\mathrm{H} 2$ ) and one cold stream (C1). Figure 6 shows a picture of the physical pilot HEN used in this work.

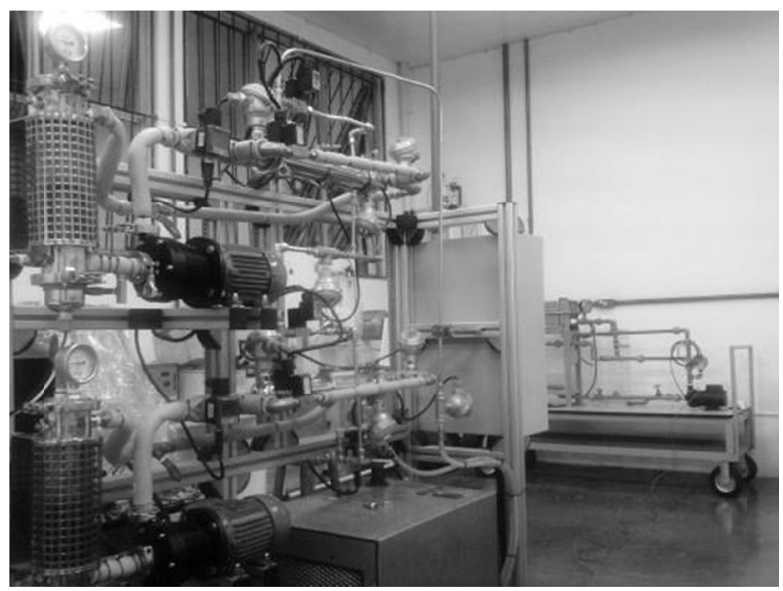

Figure 6: Experimental setup.

The manipulated inputs, controlled outputs and disturbance variables of the HEN to perform the analyses are listed below:

\section{Controlled variables:}

- $T C_{\text {OUT1 }}$ : intermediate cold stream temperature

- $T C_{\text {OUT2 }}$ : cold stream output temperature

Manipulated variables:

- $f c_{1}$ : bypass valve position, heat exchanger 1

- $f c_{2}$ : bypass valve position, heat exchanger 2

Disturbance variables:

- $T H_{I N 1}$ : hot stream 1 inlet temperature

- $T H_{I N 2}$ : hot stream 2 inlet temperature

- $T C_{I N}$ : cold stream inlet temperature

- $m_{C}$ : cold stream flowrate

Figure 7 presents a simplified piping and instrumentation diagram (P\&ID) for the pilot plant, where hot streams $\mathrm{H} 1$ and $\mathrm{H} 2$ are indicated by red lines whereas the cold stream $\mathrm{C} 1$ is indicated by blue ones. The basic instrumentation of the plant encompasses volumetric flowmeters (FT), temperature transmitters (TT) and pressure indicators (PI). There are also two vessels (V1 and V2) used to heat water, with electric resistances. The heat exchangers in the network, the connections, pumps (P1, P2 and P3) and vessels are made of ANSI 304 stainless steel. It is convenient to mention that the bypasses valves are controlled by a signal ranging from 0 up to $10 \mathrm{~V}$, where $0 \mathrm{~V}$ represents a valve full shut and $10 \mathrm{~V}$ a valve full open. 
Some characteristics of the shell and tube heat exchangers HE1 and HE2 used in the experimental HEN plant are presented in Table 1. Both exchangers have the same dimensions and the tubes are distributed in a triangular pitch. There is 1 pass in the tubes and 1 pass in the shell, with a heat transfer area $A$ equal to $0.1 \mathrm{~m}^{2}$.

Table 2 presents some nominal operational conditions in the HEN, for hot streams $\mathrm{H} 1$ and $\mathrm{H} 2$ and cold stream $\mathrm{C} 1$, where $m$ stands for flowrate, $T_{\text {in }}$ for supply temperatures and $T_{\text {out }}$ for target temperatures. Specific heat of hot and cold streams was considered equal to $4180 \mathrm{~J} \cdot \mathrm{kg}^{-1} \cdot \mathrm{K}^{-1}$.

Table 3 presents nominal operating conditions in the heat exchangers HE1 and HE2, including inlet and outlet temperatures of the hot and cold streams. The overall heat transfer coefficient $U$ was obtained through steady-state experiments and the same value was considered for both exchangers. It should be pointed out that the two controlled variables, $T C_{\text {OUT1 }}$ and $T C_{\text {OUT2 }}$, assume nominal operating temperatures of $28.5^{\circ} \mathrm{C}$ and $30.0^{\circ} \mathrm{C}$, respectively.
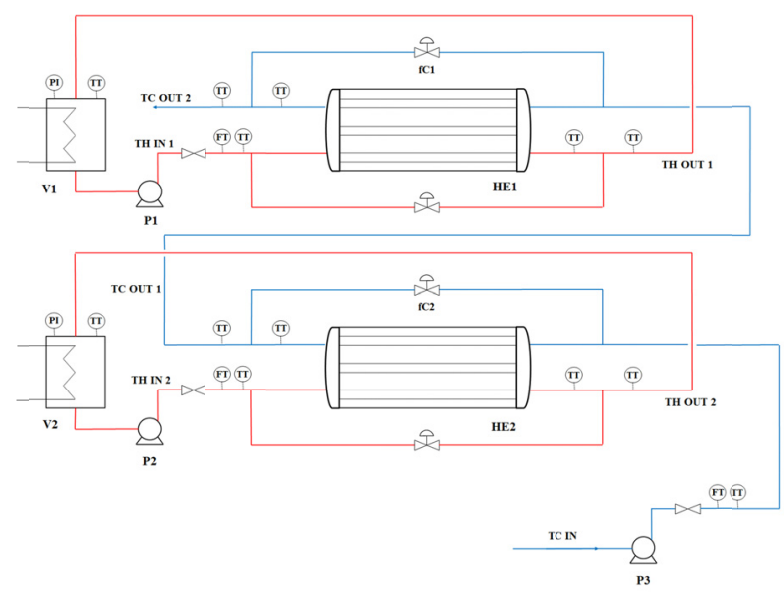

Figure 7: P\&ID of the experimental HEN.

Table 1: Heat exchanger characteristics.

\begin{tabular}{|c|l|c|c|}
\hline Variable & Description & Value & Unit \\
\hline$D_{i}$ & tube diameter & 0.006 & $\mathrm{~m}$ \\
$N_{T}$ & number of tubes & 7 & \\
$L_{T}$ & tube length & 0.750 & $\mathrm{~m}$ \\
$D_{S}$ & shell diameter & 0.060 & $\mathrm{~m}$ \\
$A$ & area & 0.1 & $\mathrm{~m}^{2}$ \\
\hline
\end{tabular}

Table 2: Stream nominal operating conditions.

\begin{tabular}{|c|c|c|c|}
\hline Stream & $\boldsymbol{m} /\left(\mathbf{k g} \cdot \mathbf{s}^{-1}\right)$ & $\boldsymbol{T}_{\text {in }} /{ }^{\mathbf{0}} \mathbf{C}$ & $\boldsymbol{T}_{\text {out }} /{ }^{\mathbf{o}} \mathbf{C}$ \\
\hline H1 & 0.154 & 55.0 & 53.4 \\
H2 & 0.154 & 55.0 & 53.3 \\
$\mathrm{C} 1$ & 0.165 & 26.9 & 30.0 \\
\hline
\end{tabular}

Table 3: Heat exchanger nominal conditions.

\begin{tabular}{|c|c|c|c|c|c|}
\hline $\begin{array}{c}\text { Heat } \\
\text { exchanger }\end{array}$ & \multicolumn{2}{|c|}{$\boldsymbol{T}_{\text {in }} /{ }^{\mathbf{o}} \mathbf{C}$} & \multicolumn{2}{|c|}{$\boldsymbol{T}_{\text {out }} /{ }^{\mathbf{o}} \mathbf{C}$} & \multirow{U}{*}{$\boldsymbol{U} / \mathbf{W} \cdot \mathbf{m}^{-\mathbf{2}} \cdot \mathbf{K}^{-1}$} \\
\hline \multirow{3}{*}{ HE1 } & hot & cold & hot & cold & \multirow{2}{*}{} \\
\cline { 2 - 5 } HE2 & 55.0 & 28.5 & 53.4 & 30.0 & 420 \\
& 55.0 & 26.9 & 53.3 & 28.5 & 420 \\
\hline
\end{tabular}

Three experimental tests were performed to evaluate the control system: two of them involving a regulatory problem, with disturbances in supply hot stream $\mathrm{H} 2$ temperature, $T H_{I N 2}$, and in cold stream flowrate $m_{C}$, and one test involving a servo problem combined with a regulatory one, with setpoint changes in controlled variables $T C_{\text {OUT1 }}$ and $T C_{\text {OUT2 }}$ and disturbances on $m_{C}$. Either in the regulatory or in the servo problem, changes were considered as step functions. The amplitudes of these step changes were chosen to avoid bypass saturation and in such a way that the variations of the controlled variables would be in the accuracy range of the measurement instruments.

\section{RESULTS AND DISCUSSION}

The application of the control structure based on LQR and applied to the HEN is shown in Figure 8, where a Luenberger observer was used in the loop for state estimation and an anti-windup subsystem was added to the integral action. The weighting $Q$ and $R$ matrices were chosen as:

$$
Q=10^{-4} \cdot[I]_{27 x 27} \quad R=[I]_{2 \times 2}
$$

where $I$ is the identity matrix. It should be pointed out that the controller has a single design parameter, which was chosen as $10^{-4}$, through a trial and error procedure, using numerical simulation. Since there is only one tuning parameter, an experimental tuning procedure could also have been used.

The target performance was to achieve a steady state decoupled system with a closed loop constant time in a range from 200 to 300 seconds. Although the system could be tuned to achieve steady state even faster than that, this amount of time is still small when compared to the dynamics of most process equipment's. Besides, the approach has some benefits: by setting a not so fast response, it may inherently prevent saturation of the actuators, provide higher robustness and reduce amplification of sensor noise.

The gain value of the anti-windup subsystem was taken as $K_{T}$ equal to 10 , a value high enough to prevent numerical problems. Simulations using the HEN 
mathematical model produced a satisfactory performance for setpoint changes and for disturbance rejection with a control effort compatible with the characteristics of the bypass valves.

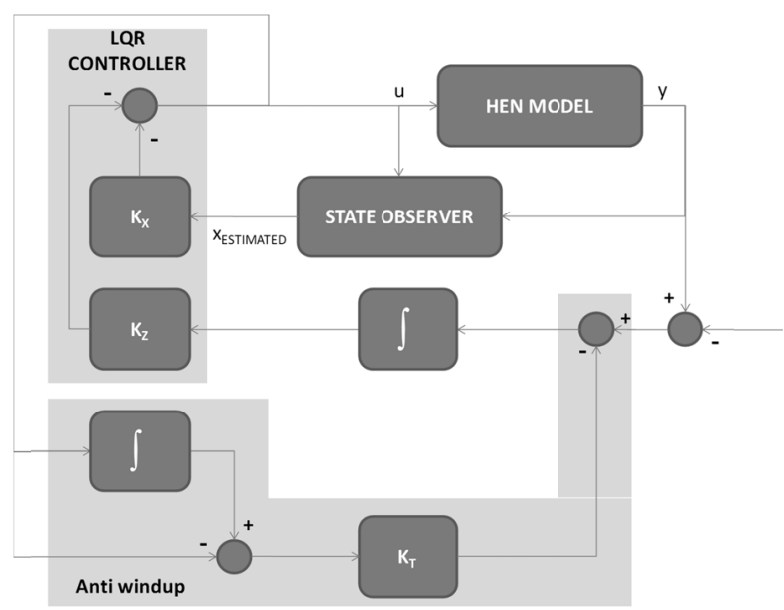

Figure 8: LQR controller structure proposed for HEN control.

As for the PID controller and the dynamic decoupler, the tuning parameters were designed according to Equation (13), with an integral gain equal to 0.04 . In the next sections the performance of common control strategies (dynamic decoupler and PID) is compared to the LQR, the proposed approach used in this work.

\section{a) Disturbance in Hot Stream Inlet Temperature $\mathrm{TH}_{I N 2}$}

To evaluate the performance of the controllers with disturbances in hot stream $\mathrm{H} 2$, a step change of $3.0^{\circ} \mathrm{C}$ was applied to $T H_{I N 2}$ at 500 s, i.e., temperature $T H_{I N 2}$ was increased to $58.0^{\circ} \mathrm{C}$. Figure 9 shows the responses of $T C_{\text {OUT1 }}$ and $T C_{\text {OUT2 }}$ for the three controllers, where the black, green and red curves are related to LQR, PID and dynamic decoupler, respectively. Figure 10 indicates the control effort of the three controllers for bypasses $f c_{1}$ and $f c_{2}$ and follows the same color patterns of Figure 9. As indicated in Figure 9, the plant was in nominal operating conditions until $500 \mathrm{~s}$, with $T C_{\text {OUT1 }}$ and $T C_{\text {OUT2 }}$ equal to $28.5^{\circ} \mathrm{C}$ and $30.0^{\circ} \mathrm{C}$, respectively. Until this time, the bypass valves were closed, as seen in Figure 10. When a disturbance in $T H_{I N 2}$ is imposed, both $T C_{O U T 1}$ and $T C_{\text {OUT2 }}$ will be affected, indicating a coupled system, as can be noted in the grid diagram of the HEN (Figure 5) and in the responses in Figure 9, at $500 \mathrm{~s}$.

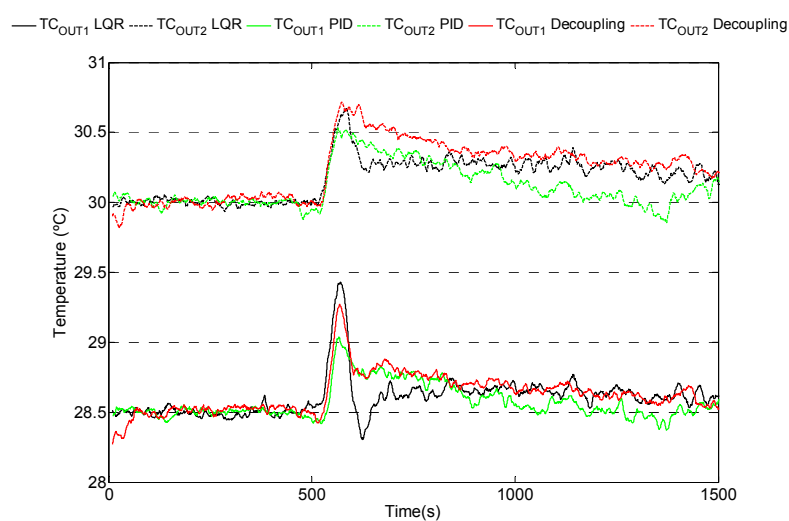

Figure 9: Plots of $T C_{\text {OUT }}$ responses under step disturbance on $T H_{I N 2}$.

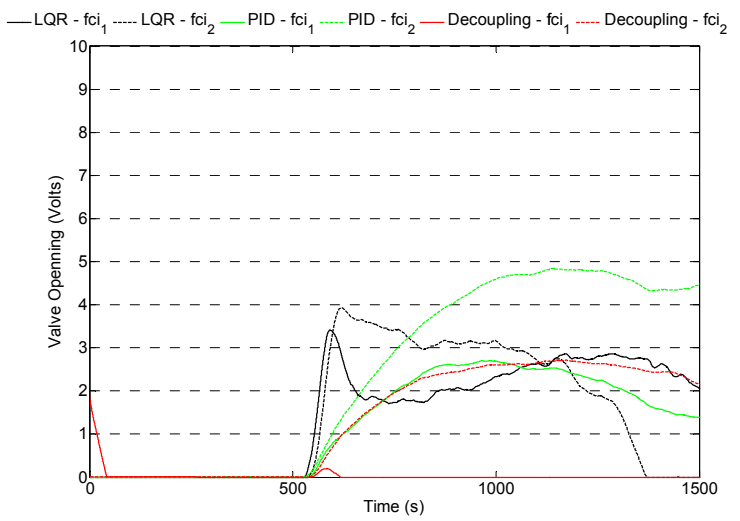

Figure 10: Control effort of bypass valves under disturbance in $T H_{I N 2}$.

It can be seen from the plots in Figure 9 that the control system pushes the controlled temperatures back to their steady-state nominal operating values, but with different transient performance, depending on which controller is used. The multi-loop PID controller opened both bypasses valves, in a smooth way, as indicated by the green lines in Figure 10. The LQR controller also put into action both bypasses, without saturation, but in a more aggressive way, as shown by the black lines in Figure 10. On the other hand, the dynamic decoupler actuated only one bypass, $f c_{2}$, and $f c_{1}$ was kept shut. This means that the dynamic decoupler tried to control both $T C_{\text {OUT1 }}$ and $T C_{\text {OUT2 }}$ by the manipulation of only one bypass. In addition to the dynamic behavior of $T C_{O U T}$ temperatures observed in Figure 9, it is important to specify a quantitative performance criterion to compare the different controllers. In this work the Integral Absolute Error or IAE (Ogunnaike and Ray, 1994) is used. Table 4 presents the controllers' performance based on IAE for LQR, PID and the dynamic decoupler. 
Table 4: Controller performance under disturbance in $\mathrm{TH}_{I N 2}$.

\begin{tabular}{|c|c|c|}
\hline \multirow{2}{*}{ LQR } & $\boldsymbol{T C}_{\text {OUT1 }}$ & $\boldsymbol{T C}_{\text {OUT2 }}$ \\
\cline { 2 - 3 } PID & 169.6 & 268.1 \\
Decoupling & 132.8 & 187.2 \\
\hline
\end{tabular}

By observing the performance of the Integral Absolute Error for the three controllers (Table 4), one can see that the PID has the best response for $T C_{\text {OUT1 }}$ and also for $T C_{\text {OUT2 }}$. However, even with a more vigorous action, the LQR controller also showed a good performance when compared to the PID. These results suggest that the tuning parameter in $Q$, which multiplies the identity matrix, and chosen as $Q$ equal to $10^{-4} \cdot I$ for the LQR design, seems to be high and leded to a faster control action when compared to the PID and dynamic decoupler.

It is worth noting that the PID and the decoupler controllers have more tuning parameters than LQR, which has only one, as considered in this work. Since this value is the unique design parameter in the controller, it is simpler to tune than the PID and the dynamic decoupler.

\section{b) Servo and Regulatory Problem}

To evaluate the servo problem in the experimental HEN, the setpoints of $T C_{\text {OUT1 }}$ and $T C_{\text {OUT2 }}$ were reduced $0.5^{\circ} \mathrm{C}$ at a time $t$ equal to $500 \mathrm{~s}$, i.e., $T C_{\text {OUT1 }}$ and $T C_{\text {OUT2 }}$ were set to $28.0^{\circ} \mathrm{C}$ and $29.5^{\circ} \mathrm{C}$, respectively. Besides, to evaluate the servo problem and also the regulatory problem, an increase in cold stream flowrate $m_{C}$ of $0.010 \mathrm{~kg} / \mathrm{s}$ was imposed at $1000 \mathrm{~s}$. Figure 11 shows the responses of $T C_{\text {OUT1 }}$ and $T C_{\text {OUT2 }}$, where the black, green and red curves are related to LQR, PID and dynamic decoupler, respectively. Figure 12 indicates the control effort of the three controllers.

By observing Figures 11 and 12, one can see that until $500 \mathrm{~s}$ the plant was under nominal operating conditions, with $T C_{\text {OUT1 }}$ and $T C_{\text {OUT2 }}$ equal to $28.5^{\circ} \mathrm{C}$ and $30.0^{\circ} \mathrm{C}$, and the bypasses valves completely closed.

After the setpoint change at $500 \mathrm{~s}$, the plots in Figures 11 and 12 suggest a relatively smooth performance for setpoint tracking, as well as for disturbance rejection, which was imposed at $1000 \mathrm{~s}$.

This behavior is quantitatively indicated in Table 5 , by using the IAE criteria. Since there is a setpoint decrease at $500 \mathrm{~s}$, the bypass valves tend to open until $1000 \mathrm{~s}$, when an increase in $m_{C}$ takes place, disturbing both $T C_{O U T 1}$ and $T C_{O U T 2}$, and then the bypasses begin to close. It can be observed that LQR action is fast when compared to the PID and the de- coupler controller, leading to a quick setpoint tracking and also disturbance rejection. This fact can be noted in Table 5, where LQR presents the smallest IAE among the three controllers.

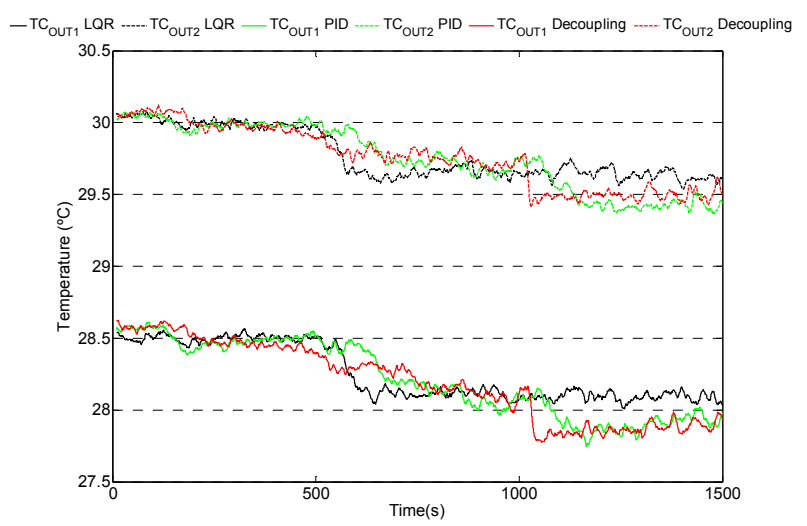

Figure 11: Plots of $T C_{\text {OUT }}$ responses under setpoint change and flowrate disturbance.

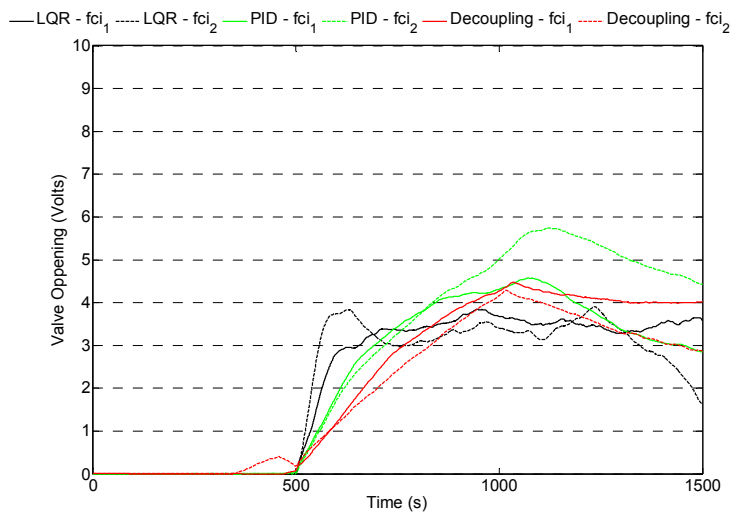

Figure 12: Control effort of bypass valves under setpoint change and flowrate disturbance.

Table 5: Controller performance under setpoint change and flowrate disturbance.

\begin{tabular}{|c|c|c|}
\hline \multirow{3}{*}{ LQR } & TCOUT1 & TCOUT2 \\
\cline { 2 - 3 } PID & 371.9 & 340.9 \\
Decoupling & 431.8 & 374.3 \\
\hline
\end{tabular}

\section{c) Disturbance in Cold Stream Flowrate $m_{C}$}

To evaluate a disturbance rejection in cold stream flowrate $m_{C}$, a variation from $0.165 \mathrm{~kg} / \mathrm{s}$ to $0.135 \mathrm{~kg} / \mathrm{s}$ was imposed at a time $t$ equal to $500 \mathrm{~s}$ and then removed at $1200 \mathrm{~s}$. The responses of $T C_{\text {OUT1 }}$ and $T C_{\text {OUT2 }}$ for the three controllers are shown in Figure 13, where the black, green and red curves are related to LQR, PID and dynamic decoupler, respectively. As previously mentioned in Table 3 , setpoints of $T C_{O U T 1}$ and 
$T C_{\text {OUT2 }}$ are equal to $28.5^{\circ} \mathrm{C}$ and $30.0^{\circ} \mathrm{C}$. Figure 14 indicates the control effort of the three controllers, using the same color patterns as in Figure 13.

It can be seen from the plots in Figure 13 and 14 that the LQR controller reacted fast, and opened both bypass valves near $500 \mathrm{~s}$, when the cold stream flowrate increased. When the flowrate in $\mathrm{C} 1$ returned to its nominal operating value, at $1200 \mathrm{~s}$, once more the LQR was fast and drove the controlled variables to $28.5^{\circ} \mathrm{C}$ and $30.0^{\circ} \mathrm{C}$. Table 6 presents the controllers performance based on IAE for LQR, PID and the dynamic decoupler.

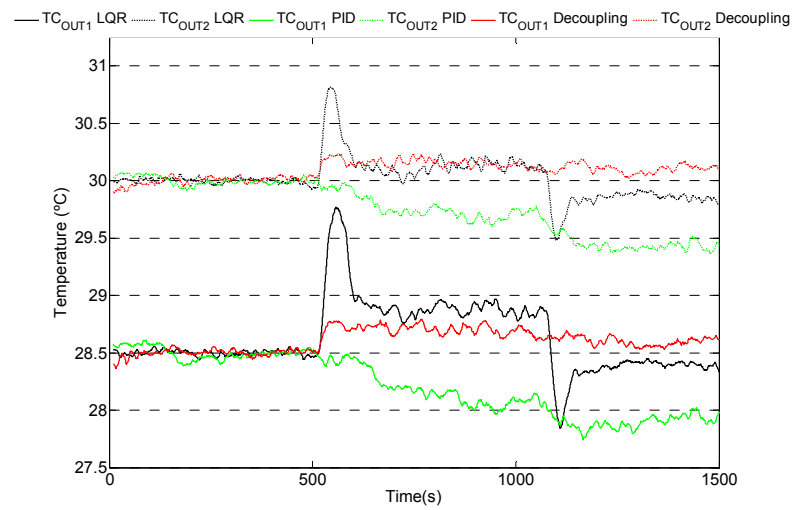

Figure 13: Plots of $T C_{\text {OUT }}$ responses under step disturbance in $m_{C}$.

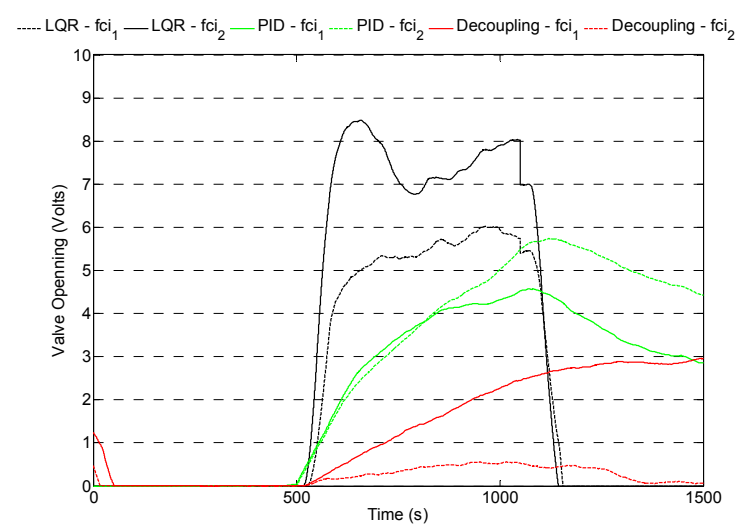

Figure 14: Control effort of bypass valves under disturbance in $m_{C}$.

Table 6: Controller performance under disturbance in $m_{C}$.

\begin{tabular}{|c|c|c|}
\hline \multirow{3}{*}{ LQR } & $\boldsymbol{T C}_{\text {OUT1 }}$ & $\boldsymbol{T C}_{\text {OUT2 }}$ \\
\cline { 2 - 3 } PID & 403.9 & 145.9 \\
Decoupling & 431.3 & 374.3 \\
\hline
\end{tabular}

For the first controlled variable, $T C_{O U T 1}$, the decoupling technique shows the best performance and the LQR is slightly better than the PID. As for $T C_{\text {OUT2 }}$, the decoupler has once more the best performance and the LQR is much better than the PID. Although the LQR was not the best option in this test, it is still a reasonable choice, due to its tuning simplicity.

\section{CONCLUSIONS}

In this work the use of a linear quadratic regulator to control heat exchanger networks was studied. This control technique is an alternative approach which presents advantages in HEN control: the LQR controller is easily designed and its performance can be even better than more common control techniques, such as PID and the dynamic decoupler. The aim of the proposed approach is not to accomplish a better performance than the one typically obtained with model predictive control techniques. On the other hand, the LQR can be designed just by using an approximate plant model and only one tuning parameter, which can be iteratively chosen.

The approximate dynamic plant model used in the design of the LQR was based on energy balance equations, which were linearized and their order reduced. However, this dynamic model could also be obtained directly from plant operation, in a more straightforward way and probably with equivalent results.

The LQR was applied to a lab scale heat exchanger network, constituted by two heat exchangers with two hot and one cold stream. Manipulated variables were the bypass valve positions and the controlled outputs were outlet temperatures. The LQR tuning parameter was determined by simulation and the controller performance was compared to the PID and the dynamic decoupler. The controllers' performance was quantitatively assessed by the integral absolute error and this index showed that the LQR performed well both in regulatory and servo problems.

\section{ACKNOWLEDGEMENTS}

The authors wish to thank all the institutional support of FEI University.

\section{REFERENCES}

Aguilera, N. and Marchetti, J. L., Optimizing and controlling the operation of heat-exchanger networks. AIChE J., 44 (5), 1090-1104 (1998).

Calandranis, J. and Stephanopoulos, G., A structural approach to the design of control systems in heat 
exchanger networks. Comp. Chem. Eng., 12(7), 651-669 (1988).

Delatore, F., Cruz, J. J., Leonardi, F. and Novazzi, L. F., Multivariable Control of a Heat Exchanger with Bypasses. 11th IASTED, Cambridge, UK (2009).

Delatore, F., Cruz, J. J., Leonardi, F. and Novazzi, L. F., Multivariable Optimal Control of a Heat Exchanger Network (HEN) with Bypasses. 12th IASTED, Banff, Canada (2010)

Giovanini, L. L. and Marchetti, J. L., Low-level flexible structure control applied to heat exchanger networks. Comp. Chem. Eng., 27, 11291142 (2003).

Glemmestad, B., Mathisen, K. W. and Gundersen, T., Optimal operation of heat exchanger networks based on structural information. Comp. Chem. Eng., 20 (suppl.), S823-S828 (1996).

Glemmestad, B., Skogestad, S. and Gundersen, T., Optimal operation of heat exchanger networks. Comp. Chem. Eng., 23, 509-522 (1999).

Gonzalez, A. H., Odloak, D. and Marchetti, J. L., Predictive control applied to heat exchanger networks. Chem. Eng. Processing, 45, 661-671 (2006).

Lersbamrungsuk, V., Srinophakun, T., Narasimhan, S. and Skogestad, S., Control structure design for optimal operation of heat exchanger networks. AIChE J., 54 (1), 150-162 (2008).
Levine, W. S., The Control Handbook. 2nd Edition, CRC Press, Boca Raton (2010).

Linhoff, B., Townsend, D. W., Boland, D., Hewitt, G. F., Thomas, B. E. A., Guy, A. R. and Marsland, R. H., A User Guide on Process Integration for the Efficient Use of Energy. Warwick Printing Company Ltd., Birmingham (1982).

Luyben, W. L., Process Modeling, Simulation and Control for Chemical Engineers. McGraw-Hill, Singapore (1990).

Marselle, D. F., Morari, M. and Rudd, D. F., Design of resilient processing plants - II. Chem. Eng. Sci., 37(2), 259-270 (1982).

Mathisen, K. W., Integrated Design and Control of Heat Exchanger Network. Ph.D. Thesis, Univ. of Trondheim, Norway (1994).

Novazzi, L. F., Dynamics and Control of Heat Exchanger Networks. Ph.D. Thesis, Univ. of Campinas, Brazil (2007). (In Portuguese).

Ogunnaike, B. A. and Ray, W. H., Process Dynamics, Modeling and Control. Oxford University Press, New York (1994).

Rotea, M. A. and Marchetti, J. L., Integral control of heat-exchanger-plus-bypass systems. IEEE Int. Conf. on Control Applic., Hartford, USA (1997).

Sun, L., Luo, X., Hou, B. and Bai, Y., Bypass selection for control of heat exchanger network. Chin. J. of Chem. Eng., 21 (3), 276-284 (2013). 